\title{
Frecuencia del maltrato y homicidio infantil en Medellín (Colombia)
}

\author{
Frequency of child abuse and homicide in Medellin (Colombia)
}

\author{
Rubén Dario Arias-Pérez $\mathbb{D}^{1}$, Tatiana Espinosa-Montoya ${ }^{1}$, Brígida Montoya-Gómez ${ }^{2}$, Melissa Botero-Bernal ${ }^{3}$
}

${ }^{1}$ Corporación Universitaria Remington, Programa de Medicina, Medellín, Colombia.

${ }^{2}$ Instituto Nacional de Medicina Legal y Ciencias Forenses, Grupo de Investigación en Ciencias Forenses, Medellín, Colombia.

${ }^{3}$ Universidad CES, Programa de Psicología, Medellín, Colombia.

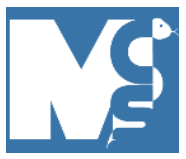

Recibido: $14 / 11 / 2020$

Revisado: 17/11/2020

Aceptado: 24/11/2020

\section{Autor correspondiente}

Melissa Botero-Bernal Universidad CES, Medellín, Colombia meli3126@gmail.com

\section{Conflictos de interés}

Los autores declaran no poseer conflictos de interés.

\section{Fuente de financiación}

El presente trabajo ha sido financiado por la Corporación Universitaria Remington, Medellín, Colombia.

Este artículo es publicado bajo una licencia de Creative Commons Reconocimiento 4.0 Internacional.

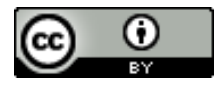

\section{RESUMEN}

Introducción: El maltrato y el homicidio infantil se destacan dentro del espectro de la violencia intrafamiliar por las consecuencias severas que generan en la salud, el desarrollo socioeconómico de una región y en la calidad de vida de los menores y sus familiares. Objetivo: Caracterizar el maltrato físico, sexual y los homicidios ejercidos hacia los niños y niñas menores de doce años, ocurridos en el contexto familiar en Medellín, Colombia, durante 2010-2015. Metodología: Estudio descriptivo, retrospectivo y transversal. Se realizó un análisis estadístico descriptivo de la ocurrencia de los eventos violentos intrafamiliares en menores de doce años atendidos por el Instituto Nacional de Medicina Legal y Ciencias Forenses en la ciudad de Medellín. Resultados: Se reportaron 3519 casos de maltrato físico y sexual infligido a menores de doce años dentro del contexto familiar. 56,9 \% de los hechos corresponden a maltrato sexual, siendo las mujeres las principales víctimas de este tipo de abuso; por su parte los hombres fueron los más afectados por maltrato físico. Los menores entre los seis y once años son los más maltratados. Se reportaron nueve casos de homicidio, de los cuales siete fueron cometidos contra mujeres y cinco de estos fueron perpetrados por el padrastro de las víctimas. Conclusiones: El maltrato infantil intrafamiliar ha sido un problema persistente en la ciudad de Medellín, y esto lo demuestra el alto número de casos registrados. No obstante, aunque los reportes de homicidio infantil han sido pocos, no se deben subestimar dada la importancia y las grandes consecuencias que esto presenta para la sociedad.

Palabras clave: Maltrato a los niños; Violencia doméstica; Abuso sexual; Abuso Físico; Homicidio

\section{ABSTRACT}

Introduction: Child abuse and homicide are highlighted in the spectrum of intrafamily violence due to the severe consequences they generate on health, the economic and social development of a region and the quality of life of children and their families. Objective: characterize the physical and sexual abuse, and homicide exercised against children under twelve years old, occurred in the family context in Medellin Colombia, during 2010-2015. Methodology: Descriptive study, retrospective and cross-sectional. It was made a descriptive statistical analysis of the occurrence of intrafamily violent events attended by the National Institute of Legal Medicine and Forensic Sciences in Medellín. Results: 3,519 cases of physical and sexual abuse inflicted on children under twelve years old within the family context were reported. Of these, $56.9 \%$ of the events correspond to sexual abuse and women are the main victims of this type of abuse; men are the most affected in physical abuse. Children between six and eleven years old are the most vulnerable. Nine cases of homicide were reported, of which seven were committed against women and five of these were perpetrated by the victims' stepfather. Conclusions: Intrafamily child abuse is a persistent problem in Medellín, and this is demonstrated by the high cases recorded. However, there have been few cases of child homicide, this does not diminish the importance and the number should not be underestimated, because of the enormous consequences they have for society.

Keywords: Child abuse; Domestic violence; Physical abuse; Sexual abuse; Homicide. 


\section{INTRODUCCIÓN}

La familia se constituye como el escenario por excelencia de aprendizaje de valores y reglas sociales. Sin embargo, también es un espacio en donde se desencadenan los mayores episodios de violencia hacia los menores de edad (1). Según la Organización Mundial de la Salud (OMS), se considera maltrato infantil a "los abusos y la desatención de que son objeto los menores de 18 años, e incluye todos los tipos de abuso físico, psicológico, sexual, desatención, negligencia y explotación comercial o de otro tipo que causen o puedan causar un daño a la salud, desarrollo o dignidad del niño, o poner en peligro su supervivencia, en el contexto de una relación de responsabilidad, confianza o poder" $(2,3)$.

En la Clasificación Internacional de las Enfermedades (CIE-10) se describen diversas modalidades de maltrato, como lo es el abuso físico, entendido como el acto de generar daño o dolor físico en el cuerpo y el abuso sexual, el cual se entiende como cualquier contacto o tentativa sexual sin consentimiento (4). Según investigaciones, los diferentes tipos de maltrato pueden generar estrés toxico $y$, repercutir de manera significativa en el adecuado desarrollo del infante (5).

Además, el maltrato se puede manifestar entre diferentes integrantes de la familia, pero son los niños y niñas los más vulnerables en las relaciones familiares (6). No obstante, esta situación pasa desapercibida en la sociedad y en la familia dado que usualmente se considera al maltrato físico como un hecho común, propio de la educación del menor y reflejo de la autoridad de los progenitores (7).

Por mucho tiempo estas prácticas fueron aceptadas y solo hasta hace poco se han reconocido como una problemática social (8); siendo el abuso sexual infantil el que ha suscitado mayor impacto social, psicológico y político en los últimos años (9).

Según datos del año 2016, aproximadamente el $20 \%$ de las mujeres en el mundo y entre el $5 \%$ al $10 \%$ de los hombres manifestaron haber sufrido abusos sexuales durante su infancia, mientras que el $23 \%$ de las personas de ambos sexos expresan maltratos físicos en su niñez. Asimismo, se calcula que cada año mueren por homicidio 41000 menores de 15 años (3).

Por su parte, en Colombia durante el año 2013, el Instituto Nacional de Medicina Legal y Ciencias Forenses de Colombia (INMLCF), notificó 68230 casos de violencia intrafamiliar, de los cuales, 9708 casos corresponden a violencia hacia niños, niñas y adolescentes, que representan el $14,3 \%$ de los casos de violencia intrafamiliar (10). Durante el año siguiente, se presentó un aumento del 7,14 \% de los hechos denunciados en comparación con el año anterior, con 10402 casos de violencia hacia niños y niñas, ubicándola en el tercer lugar de ocurrencia con respecto a los demás hechos de violencia intrafamiliar en el país (11).

A pesar de la existencia de un marco legal concerniente al maltrato infantil, las lesiones generadas por estos actos suelen ser clasificadas como accidentales por el personal asistencial, puesto que estás comúnmente ocurren en el interior de la familia, siendo difícil que los propios padres y/o cuidadores se declaren agresores y denuncien el caso. Estos aspectos generan un subregistro de la cifra real de los casos $(12,13)$ y de allí que, instituciones de orden internacional y nacional, como el Fondo de las Naciones Unidas para la Infancia (UNICEF) y el Instituto Colombiano de Bienestar Familiar (ICBF), reiteren la importancia de fomentar en la comunidad hospitalaria el interés por reconocer, notificar y llevar un registro de los casos que acuden a estos servicios, como una estrategia para visibilizar y solventar las falencias que aún existen para la identificación, diagnóstico y seguimiento de la conducta protocolaria en estos casos $(12,14,15)$.

De esta forma, el presente estudio realizado dentro del ámbito forense se planteó como objetivo caracterizar los casos de maltrato físico, sexual y homicidio hacia niños y niñas menores de doce años ocurridos dentro del contexto familiar en la ciudad de MedellínColombia durante el periodo 2010-2015.

\section{METODOLOGÍA}

La presente investigación es un estudio descriptivo, retrospectivo y transversal, que presenta la totalidad de los registros obtenidos de casos de maltrato físico, sexual, y homicidios ejercidos hacia niños y niñas menores de doce años, ocurridos dentro del contexto familiar en la ciudad de Medellín, desde enero de 2010 a diciembre de 2015.

La información que fue suministrada por el Centro de Referencia Regional sobre Violencia del INMLCF, Regional Noroccidente, encargada de la información analizada sobre indicadores de violencia y accidentalidad en la ciudad de Medellín.

No se reportó ningún dato sobre la identificación de los sujetos implicados en el estudio, conservando así, su anonimato. Se siguieron todas las pautas éticas de acuerdo con la legislación colombiana vigente y se siguieron los principios internacionales de la declaración de Helsinki. 
Para la caracterización del maltrato y los homicidios hacia los niños y niñas fueron consideradas variables sociodemográficas como edad y sexo. De igual forma se tuvieron en cuenta variables circunstanciales como la relación víctima - victimario, la clasificación del tipo o modalidad de violencia, y el año del hecho ocurrido. Cabe aclara que en la modalidad de homicidio se tuvo en cuenta el mecanismo causal más frecuente.

A la información recopilada se le aplicó un análisis estadístico descriptivo, en el cual, de acuerdo con el tipo de la variable, se calculó la distribución de frecuencia relativa y absoluta, medidas de tendencia central y de dispersión. El cálculo de la tasa de violencia para niños y niñas se realizó a partir de la información reportada por el Departamento Administrativo Nacional de Estadística (DANE), considerando el número de habitantes menores de doce años durante el periodo 2010-2015 en la ciudad de Medellín. El procesamiento y análisis de los datos se desarrolló mediante el programa Microsoft Excel 2016 y el paquete estadístico IBM SPSS Statistics versión 21.

Según las normas científicas, técnicas y administrativas para la investigación en salud del Ministerio de Salud Colombiano, el presente estudio se califica como una investigación sin riesgo.

\section{RESULTADOS}

Durante el periodo 2010-2015 en el INMLCF se reportaron 3519 casos de maltrato físico y sexual hacia niños y niñas menores de 12 años, ocurridos en el contexto familiar en la ciudad de Medellín. Cada año, en promedio, se reportaron 587 eventos [Desviación estándar $(D E)=29,5]$, expresados en una tasa especifica de 163,6 casos por cada 100000 habitantes menores de 12 años $(D E=7,9)$, aproximadamente 50 casos por mes, y entre uno y dos casos diarios de maltrato físico y sexual, a su vez el número de casos por año no posee ninguna tendencia cronológica (Tabla 1).

Asimismo, se analizaron las tasas según el sexo de las víctimas y las edades de estos, en donde se observó que, en los casos de maltrato femenino, la tasa por cada 100000 habitantes fue de 105,6; por su parte, respecto a la edad de los menores se evidenció que los de 6 años presentaron una tasa mayor respecto a las demás edades (Tabla 1).

Respecto al total de los casos reportados de maltrato el $65 \%$ de las víctimas son mujeres ( $n=2272)$. Los niños y niñas que con mayor frecuencia resultaron vulnerados tenían seis años, seguidos de aquellos con 11 años. Por su parte los menores entre 0 y 12 meses reportaron el menor número de casos (5\%). Se encuentra que la mediana de edad de todos los casos de niños y niñas violentados es de seis años [Rango intercuartílico (RIQ):4-9]. Asimismo, se evidencia una diferencia en la distribución por edad distinta en cada tipo de abuso (Tabla 1).

Al clasificar el tipo de maltrato reportado, se evidencia que los casos de maltrato sexual se presentan con mayor frecuencia que los de maltrato físico. En el periodo de estudio los reportes de eventos por maltrato sexual en Medellín representaron el $57 \%$ $(n=2004)$ de los casos (Tabla 1 ).

Respecto a la relación del agresor, según los reportes, el mayor victimario en el maltrato sexual son los otros familiares (58\%) ( $n=1172)$ y por su parte la madre es el principal victimario en el maltrato físico (38 \%) $(n=575)$ (Tabla 1$)$.

Durante el periodo de estudio se reportaron nueve casos de homicidios a niños y niñas ocurridos dentro del contexto familiar en la ciudad de Medellín, de los cuales, siete corresponden a víctimas de sexo femenino. Por otro parte, cinco de los casos están ligados a riñas pasionales entre los padres o familiares a cargo del cuidado de los menores.

Según los reportes, las víctimas presentaron una mediana de edad de cinco años (RIQ:1,5-6). Sin embargo, los niños y niñas de 6 años presentaron mayor ocurrencia del evento fatal $(33 \%)(n=3)$.

Con relación a las características de los agresores, se identifica como principales responsables a los sujetos de sexo masculino $(n=7)$. Por su parte, los mecanismos más usados para propiciar el homicidio fueron objetos contundentes $(n=3)$ y armas blancas $(n=3)$ (cortante, punzante, cortopunzante) (Tabla 2). 
TABLA 1. PRINCIPALES VARIABLES DEL MALTRATO HACIA MENORES DE 12 AÑOS EN LA CIUDAD DE MEDELLÍN, COLOMBIA ( $N=3519$ )

\begin{tabular}{|c|c|c|c|c|c|}
\hline \multicolumn{6}{|c|}{ Tipo de maltrato } \\
\hline \multicolumn{2}{|c|}{ Variables } & Físico ( $n=1515)$ & Sexual $(n=2004)$ & Total $(n=3519)$ & Tasa por 100000 Habitante \\
\hline Años & 2010 & $203(13,3)$ & $383(19,1)$ & $586(16,6)$ & 161,8 \\
\hline & 2011 & $261(17,2)$ & $362(18)$ & $623(17,7)$ & 173,1 \\
\hline & 2012 & $293(19,3)$ & $315(15,7)$ & $608(17,2)$ & 169,7 \\
\hline & 2013 & $248(16,3)$ & $289(14,4)$ & $537(15,2)$ & 150,3 \\
\hline & 2014 & $285(18,8)$ & $304(15,1)$ & $589(16,7)$ & 165,1 \\
\hline & 2015 & $225(14,8)$ & $351(17,5)$ & $576(16,3)$ & 161,6 \\
\hline \multirow[t]{2}{*}{ Sexo } & Mujer & $651(42,9)$ & $1621(80,8)$ & $2272(64,5)$ & 105,6 \\
\hline & Hombre & $864(57)$ & $383(19,1)$ & $1247(35,4)$ & 57,9 \\
\hline \multirow[t]{12}{*}{ Edad } & 0 & $70(4,6)$ & $13(0,6)$ & $83(2,4)$ & 3,8 \\
\hline & 1 & $63(4,2)$ & $37(1,8)$ & $100(2,8)$ & 4,6 \\
\hline & 2 & $73(4,8)$ & $139(6,9)$ & $212(6,0)$ & 9,8 \\
\hline & 3 & $80(5,3)$ & $242(12,1)$ & $322(9,2)$ & 14,9 \\
\hline & 4 & $108(7,1)$ & $208(10,4)$ & $316(9,0)$ & 14,6 \\
\hline & 5 & $136(9)$ & $213(10,6)$ & $349(9,9)$ & 16,2 \\
\hline & 6 & $151(10)$ & $240(12,0)$ & $391(11,1)$ & 18,1 \\
\hline & 7 & $147(9,7)$ & $194(9,7)$ & $341(9,7)$ & 15,8 \\
\hline & 8 & $158(10,4)$ & $209(10,4)$ & $367(10,4)$ & 17 \\
\hline & 9 & $154(10,2)$ & $174(8,7)$ & $328(9,3)$ & 15,2 \\
\hline & 10 & $170(11,2)$ & $160(8)$ & $330(9,4)$ & 15,3 \\
\hline & 11 & $205(13,5)$ & $175(8,7)$ & $380(10,8)$ & 17,6 \\
\hline \multirow[t]{5}{*}{ Victimario } & Padre & $532(35,1)$ & $505(25,1)$ & $1037(29,4)$ & - \\
\hline & Padrastro & $110(7,2)$ & $311(15,5)$ & $421(11,9)$ & - \\
\hline & Madrastra & $25(1,6)$ & $1(0,1)$ & $26(0,7)$ & - \\
\hline & Madre & $575(37,9)$ & $15(0,7)$ & $590(16,7)$ & - \\
\hline & Otros familiares & $273(18)$ & $1172(58,4)$ & $1445(41)$ & - \\
\hline
\end{tabular}


TABLA 2. VICTIMARIOS Y MECANISMOS USADOS EN EL HOMICIDIO DE MENORES DE 12 AÑOS DE LA CIUDAD DE MEDELLÍN, COLOMBIA SEGÚN LA DISTRIBUCIÓN DE SEXO (N=9).

\begin{tabular}{|c|c|c|c|c|}
\hline \multicolumn{2}{|l|}{ Variables } & \multicolumn{3}{|c|}{ Sexo } \\
\hline & & Hombre $(n=2)$ & Mujer ( $n=7)$ & Total $(n=9)$ \\
\hline \multirow[t]{4}{*}{ Mecanismos usados, n (\%) } & Arma blanca & - & $3(42,8)$ & $3(33,3)$ \\
\hline & Arma de fuego & $1(50)$ & - & $1(11,1)$ \\
\hline & Contundente & $1(50)$ & $2(28,5)$ & $3(33,3)$ \\
\hline & Otras & - & $2(28,5)$ & $2(22,2)$ \\
\hline \multirow[t]{4}{*}{ Victimario, n (\%) } & Madrastra & - & $1(14,2)$ & $1(11,1)$ \\
\hline & Madre & $1(50)$ & - & $1(11,1)$ \\
\hline & Padrastro & $1(50)$ & $4(57,1)$ & $5(55,5)$ \\
\hline & Otros familiares & - & $2(28,5)$ & $2(22,2)$ \\
\hline
\end{tabular}

\section{DISCUSIÓN}

Según los resultados obtenidos, los mayores casos de maltrato se dieron en los infantes entre los 6 y 11 años, estos datos coinciden con un estudio español en donde se encontró que los niños y niñas entre cinco y once años son los que mayor abuso sufren (16). A pesar de dicha concordancia, se debe aclarar que las tasas de prevalencia del maltrato en Colombia, y en general en América Latina, son mayores que las de Europa (17).

Por su parte, se evidencia que, dentro de los contextos familiares, la prevalencia según el sexo y el tipo de abuso cambia, dado que, según los resultados obtenidos, las mujeres fueron las mayores afectadas por el maltrato sexual $(80,8 \%)$ mientras que los hombres lo fueron por el maltrato físico (57\%), así mismo esto demuestra que los casos de maltrato sexual se reportan con mayor frecuencia que los de maltrato físico, resultados similares en lo observado a nivel mundial (18).

Por otra parte, en Medellín el $44 \%$ de los reportes de homicidio fueron por el subtipo de filicidio, el cual es entendido como la muerte de niños a manos de sus progenitores biológicos o adoptivos, siendo el padrastro el agresor más frecuente. Indicadores similares se han publicado en EE UU, donde la mayoría de los homicidios de menores de diez años son cometidos por el padrastro o madrastra, y en ocasiones el hecho se relaciona con represalias de pareja $(19,20)$. Se debe tener presente, que estos hechos socio patológicos son poco estudiados en América Latina.

De igual forma, resulta llamativo que el $78 \%$ de las muertes registradas en la presente investigación corresponden a casos de presunto feminicidio, que según la legislación colombiana (Ley 1761 de 2015) (21), deben ser catalogados como la forma más extrema de violencia contra la mujer. Esto concuerda con varios estudios de EE. UU en los cuales se demuestra que las mujeres menores de edad tienen el doble de probabilidad que los hombres de ser asesinadas por miembros de su familia. Asimismo, una revisión hecha en 44 países, presenta como principal víctima de homicidio infantil a las mujeres, hechos perpetrados principalmente por sus familiares o conocidos $(6,22)$.

Cabe aclarar, que a pesar de que en este estudio se observaron pocos casos de homicidio infantil, no se debe subestimar la cifra, dado que las implicaciones del maltrato y el homicidio infantil no recaen única y exclusivamente sobre las víctimas de esta violencia, pues sus consecuencias trascienden el núcleo familiar y representan pérdidas irreparables no solo para las familias sino también para la sociedad

Por otra parte, los diferentes resultados obtenidos en el presente estudio no se desvían de lo ocurrido en la población mundial, esto a pesar de las diferencias culturales y normativas (23). Sin embargo, en este estudio se logra evidenciar que los niños y niñas más pequeños (menores de 4 años) son las principales víctimas del maltrato sexual en la violencia intrafamiliar en la ciudad de Medellín, mientras que aquellos con más edad (mayores de 9 años) son los más afectados por el abuso físico, estas cifras se distancian de lo observado a nivel mundial, dado que según estadísticas de la OMS esto ocurre de manera contraria (23).

Estos actos de abuso son motivo de preocupación puesto que son prevenibles si se actúa de manera eficaz y oportuna, y usualmente no corresponden a hechos aislados. No obstante, para las víctimas por su condición de menores de edad suele ser difícil buscar 
ayuda y protección $(24,25)$. Entre los distintos factores de riesgo asociados al mantenimiento de esta problemática, se destaca el bajo desarrollo económico, el cual a su vez es consecuencia del maltrato y homicidio infantil, lo cual genera mayor desigualdad social $(17,24)$.

Por otra parte, la infancia es un periodo crucial para el adecuado desarrollo del ser humano, dado que en este periodo el cerebro tiene mayor plasticidad, lo cual permite que este órgano sea moldeado con facilidad por experiencias tanto de índole positivo como negativo (6). Según investigaciones, cualquier interferencia en esta etapa puede ocasionar diferentes consecuencias en la vida y el desarrollo de la persona, tanto a corto como a largo plazo (26), dentro de estas consecuencias se hallan deficiencias posteriores en el comportamiento, aprendizaje, bienestar físico y mental (27).

Por consiguiente es importante desarrollar políticas públicas encaminadas a los principios básicos de la primera infancia dado que un buen desarrollo en la infancia aportaría a tener una base más sólida dentro de la sociedad; asimismo, la responsabilidad sobre el adecuado desarrollo infantil no solo recae en los padres, sino también en el estado y la sociedad, puesto que estas están en constante relación, y por ende se deben articular y cumplir medidas de forma transversal para que así las políticas públicas basadas en la infancia se cumplan debidamente y estas puedan ayudar en el desarrollo socioeconómico de la comunidad y de cada uno de los sujetos implicados en ella $(5,27)$.

En este sentido, se hace el llamado a los profesionales de la salud, para que identifiquen oportunamente estos casos, considerando que tienen ante este problema una doble responsabilidad; por un lado, la legislación actual establece el compromiso de notificar el caso ante la sospecha de maltrato infantil; y por otro, existe una obligación moral, como se pone de manifiesto en estudios prospectivos sobre casos de maltrato, según la cual los profesionales de salud no tomaron las medidas para proteger a víctimas potenciales, y como consecuencia, muchos niños maltratados fueron víctimas de nuevo o sufrieron secuelas permanentes, y de mayor gravedad $(13,17,28)$.

Si bien las cifras presentadas en esta investigación son un acercamiento a la dinámica de ocurrencia de la problemática, se reconoce que pueden no ser las cifras reales, en lo que respecta a la ocurrencia del maltrato físico y sexual del cual son víctimas los niños y niñas, puesto que los registros están supeditados a la denuncia del hecho y a la valoración médico legal por parte del INMLCF. Así mismo, esta investigación tuvo varias limitaciones, una de estas es que cierta información no pudo ser tomada en cuenta puesto que esta no fue especifica o no fue dada por parte del INMLCF, por lo tanto, la forma en cómo se recolectaron los datos fue un limitante para poder realizar un análisis exhaustivo de los resultados y poder observar la realidad de la problemática, a su vez, tanto el tiempo de toma de los datos como la descripción de hechos que se dieron en una sola región del país, son factores que pueden no reflejar la realidad actual del fenómeno, por lo tanto es necesario nuevas investigaciones que contengan referencias más actualizadas en donde se puede ver si la tendencia vista en esta investigación permanece, esto con el fin de poder tener mayor información respecto al hecho real para así pensar en medidas que me ayuden a mitigar la problemática. A pesar de lo anterior, la fortaleza de esta investigación es la calidad y confianza de la información presentada, en donde las cifras dejan claro, que el maltrato infantil intrafamiliar en la ciudad de Medellín se constituye como un problema grave, que se repite constantemente y el cual se comporta de manera similar en Colombia y en América latina $(1,6)$.

En conclusión, a pesar de la existencia de los planes y programas para reducir las tasas de morbimortalidad asociadas al maltrato infantil en las grandes ciudades como Medellín, se sigue presentando esta problemática de manera frecuente. Esto resalta la importancia de realizar investigaciones multidisciplinarias que aporten una mejor comprensión de la problemática, dado que esto supone la base para que a través de políticas públicas se planifique e intervenga el fenómeno de manera adecuada, fortaleciendo a su vez herramientas en el personal de la salud y operadores de la justicia que permitan la identificación y registro de la problemática de manera precoz.

\section{AGRADECIMIENTOS}

Expresamos nuestra gratitud a la Corporación Universitaria Remington y al Instituto Nacional de Medicina Legal y Ciencias Forenses, Regional Noroccidente, por el apoyo para realizar esta investigación

\section{REFERENCIAS}

1. Instituto Nacional de Medicina Legal y Ciencias Forenses Regional Noroccidente CUR. Radiografía de la violencia regional: Retos y alientos. 1.a ed. Remington FE, editor. Medellin; 2016.

2. Organización Mundial de la Salud. Prevención del maltrato infantil: Qué hacer, y cómo obtener evidencias. Organización Mundial de la Salud. 2009. URL.

3. Fondo de las Naciones Unidas para la Infancia (UNICEF). 
Estado Mundial de la Infancia 2016. Unicef. New York; 2016. 184 p.

4. Gutiérrez-Vega I, Acosta-Ayerbe A. La violencia contra niños y niñas: un problema global de abordaje local, mediante la IAP. Rev Latinoam Cienc Soc Niñez Juv. 2013;11(1):261-72. $\underline{U R L}$.

5. Shonkoff JP. Building a New Biodevelopmental Framework to Guide the Future of Early Childhood Policy. Child Development.2010;81(1):357-67.

https://doi.org/10.1111/j.1467-8624.2009.01399.x

6. Organización de los Estados Americano. Informe sobre el castigo corporal y los derechos humanos de las niñas, niños y adolescentes. OEA, editor. 2009. 46 p.

7. Arturo Loredo-Abdalá. Maltrato infantil: su conocimiento, atención y difusión en tres hospitales pediátricos de México. Bol Méd Hosp Infant México.2016;73(4):219-27. https://doi.org/10.1016/j.bmhimx.2016.03.004

8. DuPre DP, Sites J. Investigation and Evidence Collection. En: Child Abuse Investigation Field Guide. Elsevier; 2015. p. 57108.

9. Castro Natalia. Pensando en los más pequeños "detección de medidas de prevención de abuso sexual infantil en el nivel inicial". Universidad Nacional De La Patagonia San Juan Bosco; 2013.

10. Instituto Nacional de Medicina Legal y Ciencias Forenses Grupo Centro de Referencia Nacional sobre Violencia. Comportamiento de la violencia intrafamiliar, Colombia, 2013. En: Forensis 2013. Colombia: Instituto Nacional de Medicina Legal y Ciencias Forenses; 2014. p. 335-420.

11. Instituto Nacional de Medicina Legal y Ciencias Forenses Grupo Centro de Referencia Nacional sobre Violencia. Forensis 2014, Datos para la vida. 2015;16(1):319-50. URL.

12. Fondo de las Naciones Unidas para la Infancia. Maltrato infantil: una dolorosa realidad puertas adentro. 2009.

13. Petska HW, Gordon JB, Jablonski D, Sheets LK. The Intersection of Medical Child Abuse and Medical Complexity. Pediatr Clin North Am. 2017;64(1):253-64. https://doi.org/10.1016/i.pcl.2016.08.016

14. Instituto Colombiano de Bienestar Familiar. Caracterización del maltrato infantil en Colombia: Una aproximación en cifras. Obs Bienestar Niñez. 2013;1(7):16. URL.

15. Lago Barney G, Rojas Guerrero G, Posada Vidales A, Montúfar Neira M. Síndrome de maltrato infantil. Precop SPC. 2006;2:32-53. URL.

16. Abasolo Telleria AE. Descriptive study of the type of abuse suffered by minors evaluated in the Integral Forensic Evaluation Unit of Bilbao, Spain. Span J Leg Med. 2019;45(1):4-11.

https://dx.doi.org/10.1016/j.remle.2018.04.002

17. Fondo de las Naciones Unidas para la Infancia (UNICEF). Ocultos a plena luz, un análisis estadístico de la violencia contra los niños [Internet]. Unicef. 2013. Disponible en: https://www.unicef.org/ecuador/ocultos-a-plena-luz.pdf

18. World Health Organization. Child maltreatment. Geneva; 2016. URL.

19. Ruddle A, Pina A, Vasquez E. Domestic violence offending behaviors: A review of the literature examining childhood exposure, implicit theories, trait aggression and anger rumination as predictive factors. Aggress Violent Behav. 2017;34:154-65.

https://doi.org/10.1016/j.avb.2017.01.016

20. Pierce MC, Kaczor K, Acker D, Webb T, Brenzel A, Lorenz DJ, et al. History, injury, and psychosocial risk factor commonalities among cases of fatal and near-fatal physical child abuse. Child Abuse Negl. 2017;69:263-77. https://doi.org/10.1016/j.chiabu.2017.04.033
21. Congreso de Colombia. Ley 1761 de 2015. 2015. URL.

22. Stöckl H, Dekel B, Morris-Gehring A, Watts C, Abrahams N. Child homicide perpetrators worldwide: a systematic review. BMJ Paediatr Open.2017;1(1):e000112. http://dx.doi.org/10.1136/bmjpo-2017-000112

23. Krug EG, Dahlberg LI, Mercy JA, Zwi AB, Lozano R. Informe mundial sobre la violencia y la salud. Rev Inst Med Trop São Paulo. 2003;45(3):130-130. https://doi.org/10.1590/\$0036-46652003000300014

24. Edith M, Bustillos C, Sheriff CHE. Maltrato infantil, determinantes socioeconómicos y crecimiento económico: evidencia mundial en base a datos de panel con aplicación de grupos latentes. Inst Estud Av En Desarro - INESAD. 2014;1-29. URL.

25. Van Ryzin MJ, Dishion TJ. From antisocial behavior to violence: a model for the amplifying role of coercive joining in adolescent friendships. J Child Psychol Psychiatry. 2013;54(6):661-669. https://doi.org/10.1111/jcpp.12017

26. Boyce WT. The lifelong effects of early childhood adversity and toxic stress. Pediatr Dent. 2014;36(2):102-108. https://doi.org/10.1542/peds.2011-2663

27. Hoffman MT. The Science of Early Childhood Development Closing the Gap Between What We Know and What We Do. J Dev Behav Pediatr. 2008;29(4):261. https://doi.org/10.1097/DBP.0b013e3181833804

28. Millán S, García E, Hurtado JA, Morilla M, Sepúlveda P. Victimología infantil. Cuad Med Forense. 2006;(43-44):719. URL. 